\title{
Protective efficacy of combined use of parecoxib and dexmedetomidine on postoperative hyperalgesia and early cognitive dysfunction after laparoscopic cholecystectomy for elderly patients ${ }^{1}$
}

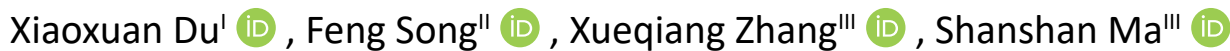

' MD, Department of Anesthesiology, the Sixth Affiliated Hospital of the Sixth Clinical Medical School, Xinjiang Medical University, Tianshan District, Urumqi, XinJiang, China. Design of the study, technical procedures, manuscript writing.

" MD, Department of Anesthesiology, the Sixth Affiliated Hospital of the Sixth Clinical Medical School, Xinjiang Medical University, Tianshan District, Urumqi, XinJiang, China. Technical procedures, analysis of data, critical revision.

I'MM, Department of Anesthesiology, the Sixth Affiliated Hospital of the Sixth Clinical Medical School, Xinjiang Medical University, Tianshan District, Urumqi, XinJiang, China. Technical procedures, analysis of data, critical revision.

\begin{abstract}
Purpose: To investigate efficacy of combined use of parecoxib and dexmedetomidine on postoperative pain and early cognitive dysfunction after laparoscopic cholecystectomy for elderly patients.

Methods: The present prospective randomized controlled study included a total of 80 patients who underwent laparoscopic cholecystectomy surgery during January 2016 to November 2017 in our hospital. All patients were randomly divided into 4 groups, the parecoxib group, the dexmedetomidine group, the parecoxib and dexmedetomidine combined group, and the control group. Demographic data and clinical data were collected. Indexes of heart rate (HR), mean arterial pressure (MAP), levels of jugular venous oxygen saturation (SjvO2) and jugular venous oxygen pressure (PjvO2) were recorded at different time points before and during the surgery. The mini-mental state examination (MMSE) score, Ramsay score and Visual Analogue Score (VAS) were measured.
\end{abstract}

Results: Levels of both $\mathrm{SjvO} 2$ and $\mathrm{PjvO} 2$ were significantly higher in parecoxib group, dexmedetomidine group and the combined group than the control group. Meanwhile, levels of both SjvO2 and PjvO2 in the combined group were the highest. VAS scores were significantly lower in the combined group than all other groups, and total patient controlled intravenous analgesia (PCIA) pressing times within $48 \mathrm{~h}$ after surgery were the lowest in the combined group. Both Ramsay and MMSE scores were the highest in the combined group compared with other groups, while were the lowest in the control group.

Conclusion: The combined use of parecoxib and dexmedetomidine could reduce the postoperative pain and improve the postoperative sedation and cognitive conditions of patients after laparoscopic cholecystectomy.

Key words: Dexmedetomidine. Pain. Cholecystectomy, Laparoscopic. 


\section{- Introduction}

Laparoscopic cholecystectomy is widely accepted as the standard operation for benign gallbladder disease ${ }^{1}$, including gallstones ${ }^{2}$, acute or chronic cholecystitis ${ }^{3,4}$ and biliary pancreatitis ${ }^{5}$. Despite the well-known advantages of laparoscopic cholecystectomy compared to open cholecystectomy, such as being a safer, more effective procedure, with less complications ${ }^{6}$, the postoperative pain and cognitive dysfunction (POCD) are still two major problems influencing patients' recovery ${ }^{7,8}$.

Parecoxib, a highly selective COX-2 inhibitor, is reported to be used in the control of postoperative pain ${ }^{9,10}$. Studies show that Parecoxib could be used in postoperative pain control in many surgeries such as laparoscopic surgeries ${ }^{11}$, total hip arthroplasty ${ }^{12}$, and cancer related operations ${ }^{13}$. Dexmedetomidine (DEX), a kind of $\alpha 2$ - adrenergic receptor agonist with sedative, analgesic, and anxiolytic properties has been applied for operative anesthesia, postoperative care, especially in mechanical ventilation and/or sedation-dependent procedures as a sole sedative or as an adjunct drug. Previous studies showed that dexmedetomidine can be used in surgery of laparoscopic cholecystectomy and could reduce postoperative pain ${ }^{14}$. However, to our best knowledge, few studies focused on efficacy of the combined use of parecoxib and dexmedetomidine on postoperative pain and early cognitive dysfunction after laparoscopic cholecystectomy for elderly patients.

In the present study, we aimed to investigate whether the combined use of parecoxib and dexmedetomidine could improve postoperative pain and early cognitive dysfunction after laparoscopic cholecystectomy for elderly patients. This study might give more clinical evidence for the application of parecoxib and dexmedetomidine in postoperative treatment of elderly patients under laparoscopic cholecystectomy.

\section{- Methods}

The present study was approved by the ethics committee of the Sixth Affiliated Hospital of the Sixth Clinical Medical School of Xinjiang Medical University.

This prospective randomized controlled single blinded study included a total of 80 patients who underwent laparoscopic cholecystectomy surgery from January 2016 to November 2017 in our hospital. All the patients who met the inclusion criteria were consecutively enrolled during the period. All patients were diagnosed as grade I II according to the American Association of Anesthesiologists (ASA) and were older than 65 years with a mean age of $69.2 \pm 4.3$ year.
The following patients were excluded: patients with preoperative cognitive dysfunction; patients with psychosis; patients with severe cardiac, lung, liver, renal or other system diseases. Patients were also excluded if the surgery time was longer than $1 \mathrm{~h}$, or the surgery could not be accomplished successfully and turned to laparotomy operation. Informed consent was obtained from all patients.

\section{Treatment and analgesia strategies}

All patients were randomly divided into 4 groups with 20 cases in each according to a computer generated randomization list run by the hospital pharmacist, 1) the parecoxib group, with preintravenous injection of $40 \mathrm{mg}$ parecoxib $30 \mathrm{~min}$ before the surgery; 2) the dexmedetomidine group, in which the patients were treated with $0.6 \mu \mathrm{g} / \mathrm{kg} / \mathrm{h}$ dexmedetomidine by intravenous injection after anesthesia induction to the end of the surgery; 3 ) the combined-group, in which patients were treated with both pre-intravenous injection of $40 \mathrm{mg}$ parecoxib and $0.6 \mu \mathrm{g} / \mathrm{kg} / \mathrm{h}$ dexmedetomidine by intravenous injection after anesthesia induction to the end of the surgery; 4) the control group, in which patients were treated with equal amount of physiological saline. The surgery strategy for all patients followed the same protocol and was conducted by the same team.

For analgesia strategies, the pre-treatment of parecoxib was performed by pre- intravenous injection with $100 \mathrm{ml}$ normal saline with $40 \mathrm{mg}$ parecoxib $^{15}$. The analgesia induction was conducted by intravenous injection of $1 \sim 2 \mathrm{mg} / \mathrm{kg}$ propofol, $0.15 \mathrm{mg} / \mathrm{kg}$ cisatracurium and $2-4 \mu \mathrm{g} / \mathrm{kg}$ fentanyl citrate followed with endotracheal intubation and mechanical ventilation. For all groups, the anesthesia maintenance was conducted using sevoflurane inhalation anesthesia. For the dexmedetomidine and the combined group, 0.6 $\mu \mathrm{g} / \mathrm{kg} / \mathrm{h}$ dexmedetomidine was kept by intravenous injection until the end of the surgery. For the control group, $0.9 \% \mathrm{NaCl}$ was given under $0.6 \mu \mathrm{g} / \mathrm{kg} / \mathrm{h}$. The postoperative patient-controlled intravenous analgesia pump (PCIA) was used for all patients. The constitution of drugs in patient-controlled intravenous analgesia pump (automatic electronic drug injection pump ZZB- II type, Jiangsu AI Peng Medical Equipment Co., Ltd., China) was: tartaric acid Bhutto $6 \mathrm{mg}+$ sufentanil citrate $0.02 \mathrm{ug} / \mathrm{kg} / \mathrm{h}$ $+0.9 \%$ physiological saline $100 \mathrm{ml}$.

\section{Data collection and measurement}

Demographic data like age, gender, body mass index (BMI), and clinical data including ASA stage, pathological type and mean operative time were 
collected. Indexes of heart rate (HR) and mean arterial pressure (MAP) were all recorded. Levels of jugular venous oxygen saturation (SjvO2) and jugular venous oxygen pressure (PjvO2) were measured by blood gas analysis using a blood gas analyzer (GEM Premier3000, Instrumentation Laboratory, MA, USA) at different time points as follows: T0 entering the surgery room, T1 trachea cannula, T2 tracheal extubation, T3 10 min after tracheal extubation. The mini-mental state examination (MMSE) score and Ramsay score were measured at $12 \mathrm{~h}, 24 \mathrm{~h}$, and $48 \mathrm{~h}$ after surgery and Visual Analogue Score (VAS) was measured at $4 \mathrm{~h}, 12$ $\mathrm{h}, 24 \mathrm{~h}$, and $48 \mathrm{~h}$ after surgery. The PCIA pressing time within $48 \mathrm{~h}$ and complications within 3 months after surgery were also recorded.

\section{Statistical analysis}

The measurement data was expressed by mean \pm SD. Counting materials were compared using Chi square test. Comparisons among three or more groups were conducted using one-way analysis of variance (ANOVA) followed by Tukey post hoc test. It was considered to be statistically significant when $P$-value was less than 0.05 . All calculations were made using SPSS 20.0.

\section{Results}

\section{Basic clinical information for all patients}

The present study selected a total of 158 patients. Among all patients, 63 patients didn't meet the inclusion criteria, 8 patients refused to participate and 7 patients were excluded because the surgery time was longer than $1 \mathrm{~h}$, or the surgery could not be accomplished successfully and turned to laparotomy operation. Finally, 80 elderly patients were included and analyzed. The flow chart is shown in Figure 1. Among the patients, 24 (30\%) patients were diagnosed as symptomatic gallstones, 36 (45\%) patients were diagnosed as acute or chronic cholecystitis and 20 (25\%) patients were diagnosed as biliary pancreatitis. No significant difference was found among different groups of patients (Table 1).

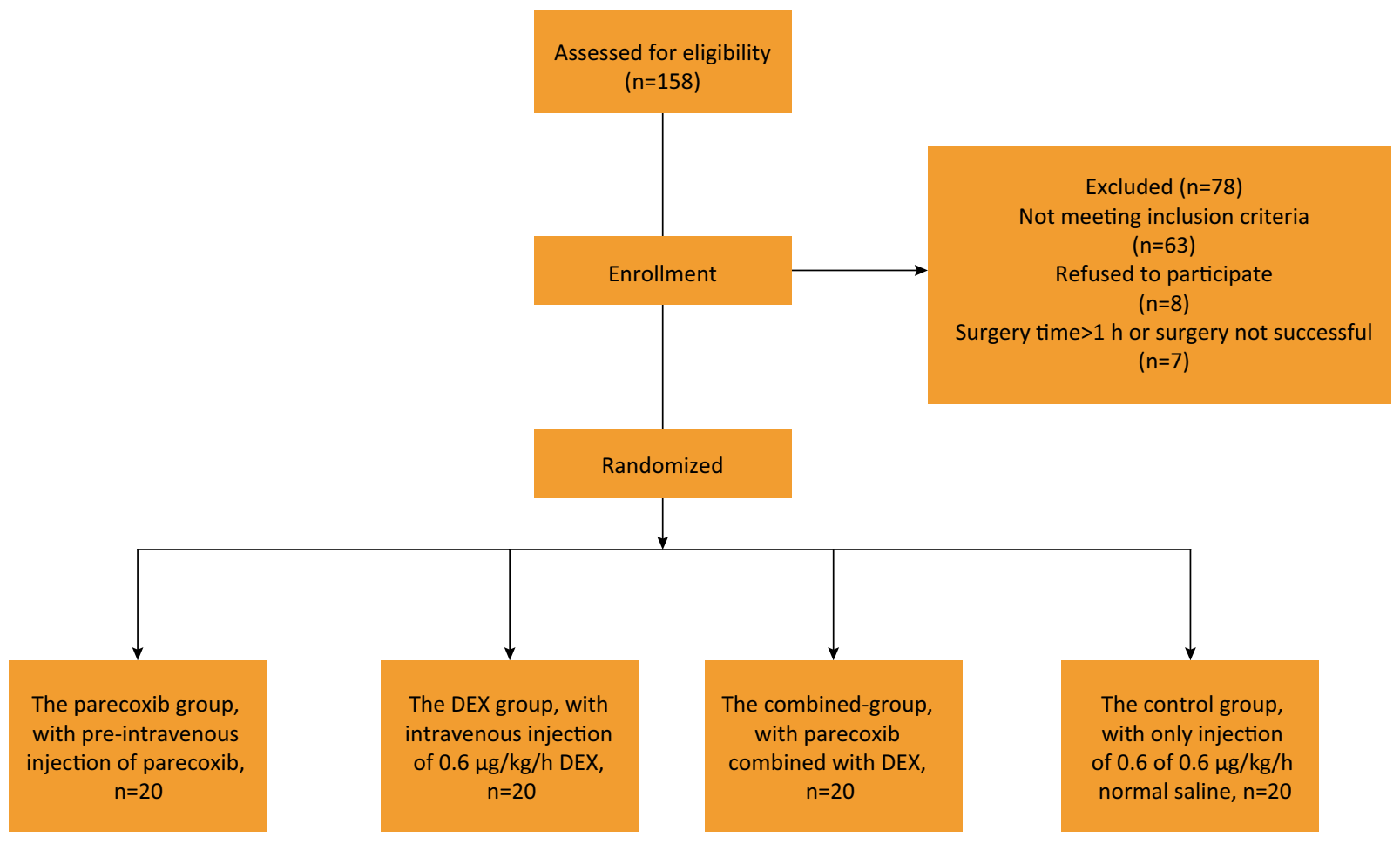

Figure 1 - Flow chart of the present study. 
Table 1 - Basic characteristics of all patients.

\begin{tabular}{|c|c|c|c|c|}
\hline Variables & Parecoxib $\mathbf{n}=\mathbf{2 0}$ & Dexmedetomidine $n=20$ & Combined $\mathbf{n}=\mathbf{2 0}$ & Control $\mathbf{n}=\mathbf{2 0}$ \\
\hline Age, year & $67.2 \pm 11.4$ & $69.3 \pm 12.5$ & $70.4 \pm 14.7$ & $68.7 \pm 13.5$ \\
\hline Gender, male: female & $12: 8$ & $11: 10$ & $13: 9$ & $11: 6$ \\
\hline \multicolumn{5}{|l|}{ ASA, $n(\%)$} \\
\hline I & $6(30)$ & $7(35)$ & $7(35)$ & $5(25)$ \\
\hline II & $14(70)$ & $13(65)$ & $13(65)$ & $15(75)$ \\
\hline Surgery duration, min & $47.5 \pm 14.8$ & $49.1 \pm 11.2$ & $46.3 \pm 10.9$ & $47.4 \pm 12.7$ \\
\hline \multicolumn{5}{|l|}{ Pathological type, n (\%) } \\
\hline Gallstones & $8(40)$ & $5(25)$ & $6(30)$ & $5(25)$ \\
\hline Cholecystitis & $7(35)$ & $9(45)$ & $10(50)$ & $10(50)$ \\
\hline Biliary pancreatitis & $5(25)$ & $6(30)$ & $4(20)$ & $5(25)$ \\
\hline
\end{tabular}

ASA Anesthesiologists grade

\section{Levels of HR, MAP and SjvO2, PjvO2 in different} groups of patients at different time points

As shown in Table 2, the levels of HR, MAP and SjvO2, $\mathrm{PjVO2}$ at different time points were measured and collected. Results showed that no significant difference was found for HR and MAP levels among different groups at all the time points. However, levels of both SjvO2 and PjvO2 were significantly higher in parecoxib group, dexmedetomidine group and the combined group than the control group at $\mathrm{T} 2$ and $\mathrm{T} 3$ time points $(P<0.05)$. Meanwhile, levels of both SjvO2 and PjvO2 in the combined group were the highest of all other groups at $\mathrm{T} 2$ and $\mathrm{T} 3$ time points $(\mathrm{P}<0.05)$.

Table 2 - Levels of HR, MAP and SjvO2, PjvO2 in different groups of patients at different time points.

\begin{tabular}{|c|c|c|c|c|c|}
\hline Variables & Groups $\mathbf{n}=\mathbf{2 0}$ & TO & T1 & T2 & T3 \\
\hline \multirow{4}{*}{$\mathrm{HR}$, frequency/min } & Parecoxib & $78.3 \pm 9.4$ & $79.0 \pm 8.7$ & $79.6 \pm 9.5$ & $78.3 \pm 7.3$ \\
\hline & Dexmedetomidine & $77.9 \pm 8.6$ & $78.2 \pm 6.0$ & $81.7 \pm 8.8$ & $78.6 \pm 7.9$ \\
\hline & Combined & $78.4 \pm 6.1$ & $76.8 \pm 8.3$ & $81.4 \pm 9.7$ & $79.5 \pm 8.7$ \\
\hline & Control & $79.1 \pm 8.9$ & $80.3 \pm 7.2$ & $83.9 \pm 7.2$ & $81.1 \pm 7.2$ \\
\hline \multirow{4}{*}{$\mathrm{MAP}, \mathrm{mm} \mathrm{Hg}$} & Parecoxib & $95.3 \pm 8.2$ & $87.2 \pm 8.5$ & $94.5 \pm 8.1$ & $95.7 \pm 8.3$ \\
\hline & Dexmedetomidine & $97.1 \pm 9.5$ & $86.1 \pm 7.6$ & $95.7 \pm 8.3$ & $96.4 \pm 7.5$ \\
\hline & Combined & $96.4 \pm 6.7$ & $87.5 \pm 7.8$ & $95.6 \pm 8.1$ & $95.8 \pm 6.9$ \\
\hline & Control & $96.8 \pm 7.4$ & $87.3 \pm 8.3$ & $95.3 \pm 9.0$ & $96.5 \pm 9.3$ \\
\hline \multirow{4}{*}{ SjvO2 } & Parecoxib & $60.3 \pm 7.1$ & $72.4 \pm 9.2$ & $66.3 \pm 7.9 *, \#$ & $64.3 \pm 5.6^{*, \#}$ \\
\hline & Dexmedetomidine & $61.2 \pm 8.9$ & $73.1 \pm 7.1$ & $66.1 \pm 5.2^{*, \#}$ & $64.2 \pm 5.1 *, \#$ \\
\hline & Combined & $61.7 \pm 6.0$ & $73.9 \pm 7.6$ & $71.7 \pm 6.4^{*}$ & $68.5 \pm 5.7 *$ \\
\hline & Control & $60.9 \pm 7.5$ & $72.8 \pm 6.3$ & $63.5 \pm 5.8$ & $61.0 \pm 5.5$ \\
\hline \multirow{4}{*}{$\mathrm{PjvO2}$} & Parecoxib & $40.7 \pm 5.0$ & $48.9 \pm 5.4$ & $44.7 \pm 4.5^{*, \#}$ & $42.5 \pm 2.2^{*, t}$ \\
\hline & Dexmedetomidine & $40.9 \pm 6.3$ & $48.2 \pm 5.6$ & $44.9 \pm 4.2^{*, \#}$ & $42.2 \pm 2.0^{*,+}$ \\
\hline & Combined & $41.3 \pm 5.8$ & $49.5 \pm 5.7$ & $48.3 \pm 5.1 *$ & $44.3 \pm 3.1 *$ \\
\hline & Control & $41.6 \pm 5.6$ & $49.7 \pm 6.0$ & $41.1 \pm 4.9$ & $41.0 \pm 1.6$ \\
\hline
\end{tabular}

HR heart rate, MAP mean arterial pressure, SjvO2 jugular venous oxygen saturation, PjvO2 jugular venous oxygen pressure. ${ }^{*} \mathrm{P}<0.05$, compared with the control group; ${ }^{\mathrm{P}<0.05}$ compared with the combined group. 


\section{Pain conditions of different groups of patients by VAS scores and PCIA pressing times}

Further pain condition analysis showed in patients of parecoxib group, dexmedetomidine group and the combined group, VAS scores were significantly lower than the control group at all the time points except for $48 \mathrm{~h}$ after surgery $(P<0.05$, Table 3$)$. Moreover, VAS scores were significantly lower in the combined group than all other groups except for $48 \mathrm{~h}$ after surgery $(P<0.05)$. Similarly, total $\mathrm{PCIA}$ pressing times within $48 \mathrm{~h}$ after surgery were the lowest in the combined group $(P<0.05)$, while the highest in the control group $(P<0.05)$. These results suggested combined use of parecoxib and dexmedetomidine could significantly reduce the postoperative pain of patients after laparoscopic cholecystectomy.

Table 3 - Pain conditions of different groups of patients by VAS scores and PCIA pressing times.

\begin{tabular}{|c|c|c|c|c|c|}
\hline Variables & Time points & Parecoxib & Dexmedetomidine & Combined & Control \\
\hline \multirow{4}{*}{ VAS } & $4 \mathrm{~h}$ & $3.7 \pm 1.7^{c, d}$ & $3.9 \pm 1.3^{c, d}$ & $2.4 \pm 1.0^{\mathrm{a}, \mathrm{b}, \mathrm{d}}$ & $5.8 \pm 2.3^{a, b, c}$ \\
\hline & $12 \mathrm{~h}$ & $2.9 \pm 0.9^{c, d}$ & $3.0 \pm 1.2^{\mathrm{c}, \mathrm{d}}$ & $1.8 \pm 0.4^{\mathrm{a}, \mathrm{b}, \mathrm{d}}$ & $4 \cdot 6 \pm 1.7^{a, b, c}$ \\
\hline & $24 \mathrm{~h}$ & $1.9 \pm 0.2^{c, d}$ & $2.1 \pm 0.6^{\mathrm{c}, \mathrm{d}}$ & $1.4 \pm 0.1^{\mathrm{a}, \mathrm{b}, \mathrm{d}}$ & $2.8 \pm 0.8^{a, b, c}$ \\
\hline & $48 \mathrm{~h}$ & $1.1 \pm 0.1^{\mathrm{d}}$ & $1.0 \pm 0.1^{\mathrm{d}}$ & $1.1 \pm 0.1^{\mathrm{d}}$ & $1.2 \pm 0.1^{\mathrm{a}, \mathrm{b}, \mathrm{c}}$ \\
\hline PCIA & $48 \mathrm{~h}$ & $5.0 \pm 1.3^{c, d}$ & $5.1 \pm 1.9^{c, d}$ & $3.4 \pm 1.4^{\mathrm{a}, \mathrm{b}, \mathrm{d}}$ & $7.8 \pm 2.3^{a, b, c}$ \\
\hline
\end{tabular}

${ }^{a} \mathrm{P}<0.05$, compared with the Parecoxib group; ${ }^{\mathrm{b}} \mathrm{P}<0.05$, compared with $D E X$ group; ${ }^{\mathrm{c} P}<0.05$, compared with Combined group; ${ }^{d} \mathrm{P}<0.05$, compared with Control group. VAS Visual Analogue Score, PICA patient-controlled intravenous analgesia pump.

\section{Ramsay and MMSE scores and postoperative agitation of different groups of patients after surgery}

At last, the postoperative sedation and cognitive conditions were analyzed using Ramsay and MMSE scores, respectively. As shown in Table 4, both
Ramsay and MMSE scores were the highest in the combined group compared with other groups $(P<0.05)$, while were the lowest in the control group. These results indicated the combined use of parecoxib and dexmedetomidine could significantly improve the postoperative sedation and cognitive conditions of patients after laparoscopic cholecystectomy.

Table 4 - Pain conditions of different groups of patients by MMSE and Ramsay scores.

\begin{tabular}{|c|c|c|c|c|c|}
\hline Variables & Time points & Parecoxib & Dexmedetomidine & Combined & Control \\
\hline \multirow{4}{*}{ MMSE } & Before & $26.3 \pm 1.4$ & $26.5 \pm 1.7$ & $27.0 \pm 1.5$ & $26.8 \pm 1.5$ \\
\hline & $12 \mathrm{~h}$ & $22.6 \pm 1.6^{c, d}$ & $22.4 \pm 1.9^{c, d}$ & $24.5 \pm 1.6^{a, b, d}$ & $19.4 \pm 1.8^{\mathrm{a}, \mathrm{b}, \mathrm{c}}$ \\
\hline & $24 \mathrm{~h}$ & $24.0 \pm 1.4^{c, d}$ & $23.7 \pm 1.3^{c, d}$ & $25.7 \pm 1.8^{\mathrm{a}, \mathrm{b}, \mathrm{d}}$ & $22.3 \pm 1.4^{a, b, c}$ \\
\hline & $48 \mathrm{~h}$ & $25.1 \pm 1.5^{\mathrm{d}}$ & $25.0 \pm 1.5^{d}$ & $26.9 \pm 1.9^{d}$ & $24.1 \pm 1.1^{\mathrm{a}, \mathrm{b}, \mathrm{c}}$ \\
\hline \multirow{3}{*}{ Ramsay } & $12 \mathrm{~h}$ & $2.4 \pm 0.7^{c, d}$ & $2.5 \pm 0.5^{c, d}$ & $2.8 \pm 0.5^{\mathrm{a}, \mathrm{b}, \mathrm{d}}$ & $1.9 \pm 0.4^{\mathrm{a}, \mathrm{b}, \mathrm{c}}$ \\
\hline & $24 \mathrm{~h}$ & $2.3 \pm 0.6^{c, d}$ & $2.3 \pm 0.5^{c, d}$ & $2.6 \pm 0.3^{\mathrm{a}, \mathrm{b}, \mathrm{d}}$ & $2.0 \pm 0.6^{a, b, c}$ \\
\hline & $48 \mathrm{~h}$ & $2.3 \pm 0.5^{\mathrm{d}}$ & $2.3 \pm 0.6^{d}$ & $2.5 \pm 0.8^{d}$ & $2.0 \pm 0.3^{a, b, c}$ \\
\hline
\end{tabular}

${ }^{a} \mathrm{P}<0.05$, compared with the Parecoxib group; ${ }^{b} \mathrm{P}<0.05$, compared with $\mathrm{DEX}$ group; ${ }^{\mathrm{c}} \mathrm{P}<0.05$, compared with Combined group; ${ }^{\mathrm{d}} \mathrm{P}<0.05$, compared with Control group. MMSE mini-mental state examination score.

\section{- Discussion}

Though several studies have reported efficacy of parecoxib and dexmedetomidine in cholecystectomy, few of them focused on the combined use of parecoxib and dexmedetomidine. In the present study, we demonstrated that the combined use of parecoxib and dexmedetomidine could reduce postoperative pain and improve postoperative sedation and cognitive conditions of patients after laparoscopic cholecystectomy.

Efficacy of parecoxib in postoperative treatment has been reported in many surgeries, including cholecystectomy. 
Shuying et al showed intravenous parecoxib could reduce the length of stay on ambulatory for patients who underwent laparoscopic cholecystectomy ${ }^{16}$. Luo et al. ${ }^{17}$ demonstrated intravenous injection of parecoxib could provide effective analgesic effect in laparoscopic cholecystectomy and was better than flurbiprofen in terms of analgesic effect. It was also found parecoxib could reduce shoulder pain after gynecologic laparoscopy ${ }^{18}$. In the present study, we also found parecoxib could reduce the postoperative pain and improve the postoperative sedation and cognitive conditions of patients after laparoscopic cholecystectomy, which was consistent with previous researches. And the application of the combined use of dexmedetomidine could enhance the effects.

Application of dexmedetomidine in cholecystectomy has also been demonstrated in several studies. Kang et al showed dexmedetomidine administration during surgery could reduce intraoperative and post-operative secretion of cytokines and post-operative leukocyte count and CRP levels of cholecystectomy patients ${ }^{19}$. $\mathrm{Yu}$ et $a .^{20}$ demonstrated ropivacaine combined with dexmedetomidine could significantly improve the pain condition for patients after laparoscopic cholecystectomy. It was also considered dexmedetomidine could shift the Th1/Th2 cytokine balance toward Th1 in patients with surgical and anesthetic stress ${ }^{21}$. In our study, we demonstrated the combined use of parecoxib and dexmedetomidine could reduce the postoperative pain and improve the postoperative sedation and cognitive conditions of patients after laparoscopic cholecystectomy, which might provide a new method to improve postoperative recovery. The present study also has some limitations. We included a small sample size from a single hospital and deeper insights as well as more clinical evidences are still needed for the application of parecoxib and dexmedetomidine in postoperative treatment.

\section{- Conclusions}

The combined use of parecoxib and dexmedetomidine could reduce the postoperative pain and improve the postoperative sedation and cognitive conditions of patients after laparoscopic cholecystectomy. This study might give more clinical evidence for the application of parecoxib and dexmedetomidine in the postoperative treatment of elderly patients under laparoscopic cholecystectomy.

\section{- References}

1. Rosen M, Brody F, Ponsky J. Predictive factors for conversion of laparoscopic cholecystectomy. Indianjj Surg. 2013;75(2):152. Doi: 10.1007/s12262-012-0503-y.
2. Jabbari NAHM, Jangjoo A. Consequences of lost gallstones during laparoscopic cholecystectomy: a review article. Surg Laparo Endoper. 2016;26(3):183. Doi: 10.1097/ SLE.0000000000000274.

3. Wani MM. Open versus laparoscopic cholecystectomy in acute cholecystitis. Systematic review and metaanalysis. Int J Surg. 2015;18(27):196-204. Doi: 10.1016/j. ijsu.2015.04.083.

4. Igami T, Aoba T, Ebata T, Yokoyama Y, Sugawara G, Nagino M. Single-incision laparoscopic cholecystectomy for cholecystitis requiring percutaneous transhepatic gallbladder drainage. Surg Today. 2015;45(3):305-9. Doi: 10.1007/s00595-014-1003-4.

5. Lacher M YG, Muensterer OJ, Aprahamian CJ, Haricharan RN, Perger L, Bartle D, Talathi SS, Beierle EA, Anderson SA, Chen MK, Harmon CM. Laparoscopic cholecystectomy for biliary dyskinesia in children: frequency increasing. J Pediatr Surg. 2013;48(8):1716-21. Doi: 10.1016/j.jpedsurg.2012.08.036.

6. Coenye KE, Jourdain S, Mendes dcp. Laparoscopic cholecystectomy for acute cholecystitis in the elderly: a retrospective study. Hepatogastroenterology. 2005;52(52):17-21. Doi: 10.1046/j.1365-2168.1998.00708.x.

7. Geng YJ, Wu QH, Zhang RQ. Effect of propofol, sevoflurane, and isoflurane on postoperative cognitive dysfunction following laparoscopic cholecystectomy in elderly patients: a randomized controlled trial. J Clin Anesth. 2017;38:16571. Doi: 10.1016/j.jclinane.2017.02.007.

8. Han IW, Kwon OC, Oh MG, Choi YS, Lee SE. Effects of Rowachol on prevention of postcholecystectomy pain after laparoscopic cholecystectomy: prospective multicenter randomized controlled trial. HPB. 2016;18(8):664-70. Doi: 10.1016/j.hpb.2016.05.013.

9. Maxwell $M$, Nathanson $M$. Parecoxib--getting to the heart of the matter. Anaesthesia. 2010;61(9):823-5. Doi: 10.1111/j.1365-2044.2007.05005_1.x.

10. Lv N, Kong Y, Mu L, Pan T, Xie Q, Zhao M. Effect of perioperative parecoxib sodium on postoperative pain control for transcatheter arterial chemoembolization for inoperable hepatocellular carcinoma: a prospective randomized trial. Eur Radiol. 2016;26(10):3492-9. Doi: 10.1007/s00330-016-4207-8.

11. Huang JM, Lv ZT, Zhang YN, Jiang WX, Li HN, Nie MB. Efficacy and safety of postoperative pain relief by parecoxib injection after laparoscopic surgeries: a systematic review and meta-analysis of randomized controlled trials. Pain Pract. 2018;18(5):597-610. Doi: 10.1111/papr.12649.

12. Camu F, Borgeat A, Heylen RJ, Viel EJ, Boye ME, Cheung RY. Parecoxib, propacetamol, and their combination for analgesia after total hip arthroplasty: a randomized noninferiority trial. Acta Anaesth Scand. 2017;61(1):99-110. Doi: 10.1111/aas.12841.

13. Kenner DJ, Sandeep B, Fullerton SL. Daily subcutaneous parecoxib injection for cancer pain: an open label pilot study. J Palliat Med. 2015;18(4):366-72. Doi: 10.1089/ jpm.2014.0249.

14. Bielka K, Kuchyn I, Babych V, Martycshenko K, Inozemtsev $\mathrm{O}$. Dexmedetomidine infusion as an analgesic adjuvant during laparoscopic cholecystectomy: a randomized controlled study. BMC Anesthesiol. 2018;18(1):44. Doi: 10.1186/s12871-018-0508-6. 
15. Pandazi A, Kapota E, Matsota P, Paraskevopoulou P, Dervenis C, Kostopanagiotou G. Preincisional versus postincisional administration of parecoxib in colorectal surgery: effect on postoperative pain control and cytokine response. A randomized clinical trial. World J Surg. 2010;34(10):24639. Doi: 10.1111/j.1365-2044.2007.05005_1.x.

16. Li S, Wang X, Liang P, Zhu T, Lu Z, Zhao L. Preoperative intravenous parecoxib reduces length of stay on ambulatory laparoscopic cholecystectomy. Int J Surg. 2014;12(5):464-8. Doi: 10.1016/j.ijsu.2014.03.013.

17. Luo X, Lv F, Peng M. Analgesic effect of different dosage of Flurbiprofen axetil in laparoscopic cholecystectomy in comparison with other analgesic drugs. Pak J Pharm Sci. 2017 Sep;30(5(Special)):1895-8. PMID: 29084663.

18. Zhang H, Shu H, Yang L, Cao M, Zhang J, Liu K, Xiao L, Zhang $X$. Multiple-, but not single-, dose of parecoxib reduces shoulder pain after gynecologic laparoscopy. Int J Med Sci. 2012;9(9):757-65. Doi: 10.7150/ijms.4916.

19. Kang SH, Kim YS, Hong TH, Chae MS, Cho ML, Her YM, Lee J. Effects of dexmedetomidine on inflammatory responses in patients undergoing laparoscopic cholecystectomy. Acta Anaesth Scand. 2013;57(4):480-7. Doi: 10.1111/aas.12039.

20. Yu JM, Sun H, Wu C, Dong CS, Lu Y, Zhang Y. The analgesic effect of ropivacaine combined with dexmedetomidine for incision infiltration after laparoscopic cholecystectomy. Surg Laparo Endoper. 2016;26(6):449. Doi: 10.1097/ SLE.0000000000000325.

21. Kim Y, Kang SH, Hong TH, Cho ML, Han HJ, Kwon SJ, Lee J. Effects of dexmedetomidine on the ratio of $T$ helper 1 to $\mathrm{T}$ helper 2 cytokines in patients undergoing laparoscopic cholecystectomy. J Clin Anesth. 2014;26(4):281-5. doi: 10.1016/j.jclinane.2013.11.018.

\section{Correspondence:}

Xiaoxuan Du

Department of Anesthesiology, the Sixth Affiliated Hospital

the Sixth clinical Medical School, Xinjiang Medical University

Tianshan District, Urumqi, XinJiang, 830002 China

Phone: +86 0991-2661545

xiaoxuandu12@163.com

Received: May 03, 2019

Review: July 06, 2019

Accepted: Aug 04, 2019
Conflict of interest: none

Financial source: Project " $A$ new mechanism of dexmedetomidine regulating IL-17A expression in subacute phase inflammatory response to protect organs from injury" (ID 2017D01C264)

${ }^{1}$ Research performed at Department of Anesthesiology, the Sixth Affiliated Hospital of the Sixth Clinical Medical School, Xinjiang Medical University, Tianshan District, Urumqi, XinJiang, China. 